\title{
Depressive symptoms and their associated factors in heart failure patients
}

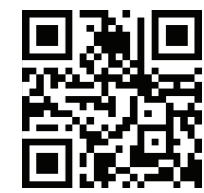

\author{
Deng-Xin He $\mathrm{a}^{\mathrm{a}, \mathrm{*}, \dagger}$, Ming-Hao Pan ${ }^{\mathrm{b}, \dagger}$ \\ aSchool of Nursing, Hubei University of Chinese Medicine, Wuhan, Hubei 430065, China \\ ${ }^{b}$ School of Medicine, Xinyang Normal University, Xinyang, Henan 464000, China
}

Received: 28 March 2021; Accepted: 28 April 2021; Published: 20 December 2021

\begin{abstract}
Objectives: Depressive symptoms are common in heart failure (HF) patients and they may exacerbate the progression of HF. Thus, identifying associations with depressive symptoms is essential to develop effective interventions to alleviate patients' depressive symptoms. Therefore, this study aimed to explore the factors related to HF patients' depressive symptoms.

Methods: Potential hospitalized patients were recruited from a university-affiliated hospital by convenience sampling. This study included $321 \mathrm{HF}$ patients who had completed information about depressive symptoms, functional capacity, social support, dispositional optimism, self-care confidence, and health literacy. Descriptive statistics were used to characterize the sample. Independent group $t$-tests and one-way analysis of variance were used to assess the difference in depressive symptoms score in demographic and clinical characteristics, while Pearson's correlation was used to assess the associations among continuous variables.

Results: The scores for functional capacity, social support, dispositional optimism, self-care confidence, and health literacy were negatively associated with the score for depressive symptoms. The interaction effect between the functional capacity and the dispositional optimism on depressive symptoms was significant.

Conclusions: Interventions targeted improving the above-mentioned factors may be beneficial to reduce depressive symptoms in HF patients.
\end{abstract}

Keywords: depressive symptoms • dispositional optimism • functional capacity • heart failure • social support

(c) Shanxi Medical Periodical Press.

\section{Introduction}

Depressive symptoms are common in patients with heart failure (HF). ${ }^{1}$ It has been found that the prevalence rates of depressive symptoms range from $24 \%$ to $42 \%$ in patients with $\mathrm{HF}^{2}$ and $58 \%$ in hospitalized older patients with $\mathrm{HF}^{3}$ Depressive symptoms are demonstrated to be associated with poor prognosis in patients with $\mathrm{HF}^{4,5}$ It was found that both depressive symptoms in baseline and worsening depressive symptoms in 1-year followup increased the risk of HF patients' death or cardiovascular hospitalization. ${ }^{6,7}$ Similarly, depressive symptoms could significantly predict increased all-cause mortality during 1-year follow-up. ${ }^{8}$ Furthermore, the hazard was found to differ over time with depressive symptoms posing a little risk for HF patients' mortality in the first year, but the greater risk in the second and third years

† Deng-Xin He and Ming-Hao Pan contribute equally to this work.

How to cite this article: He DX, Pan MH. Depressive symptoms and their associated factors in heart failure patients. Front Nurs. 2021;4:389-398. 
of follow-up. ${ }^{9}$ In addition, depressive symptoms were reported to be associated with reduced quality of life in patients with HF., ${ }^{4,5,10}$ Therefore, it is essential to understand the factors associated with depressive symptoms to develop effective interventions to relieve HF patients' depressive symptoms.

Diathesis-stress theories provide the basis for developing frameworks for investigating potential factors of depressive symptoms. ${ }^{11}$ Diathesis, which could be considered as vulnerability, includes cognitive and psychosocial factors that cause individuals to be vulnerable to depressive symptoms in response to stress. Stress is defined as "environmental events or chronic conditions that objectively threaten the physical and/or psychological health or well-being of individuals of a particular age in a particular society". ${ }^{12}$ Diathesis-stress theories suggest that diathesis and stress contribute to the development of depressive symptoms, and particular stress may interact with diathesis. Thus, some specific diatheses and stress may cause HF patients to be vulnerable to depressive symptoms. Based on diathesis-stress theories, we operationalized impaired functional capacity as potential stress, lack of social support, low dispositional optimism, low self-care confidence, and low health literacy as potential diathesis (Figure 1).

It is critical to identify the potential factors related to HF patients' depressive symptoms because this would provide referential information for the development of interventions to relieve their depressive symptoms. Thus, the current study aimed to assess the associations between potential factors and HF patients' depressive symptoms. We inferred that impaired functional capacity, low social support, low dispositional optimism, low self-care confidence, and low health literacy were associated with depressive symptoms in patients with HF and impaired functional capacity may interact with potential diatheses.

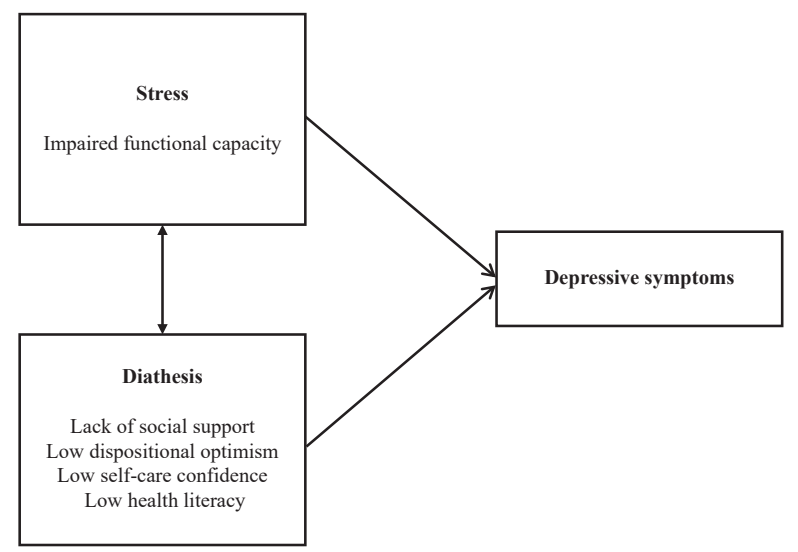

Figure 1. The conceptual model guiding this study.

\section{Methods}

\subsection{Design and procedures}

This study was designed to examine the factors associated with depressive symptoms in patients with HF. Potential patients were recruited from a universityaffiliated hospital by convenience sampling. A trained research assistant assessed whether patients were eligible and obtained written consent from eligible patients. After patients completed the questionnaires provided, the assistant checked the questionnaires to ensure accuracy and integrity.

This study conforms to the principles outlined in the Declaration of Helsinki. Researchers recruited eligible patients in three cardiovascular units of a large, university-affiliated hospital in Shandong province, China, from November 2016 to June 2017. Patients who (a) had HF diagnosed, (b) were aged 18 years, (c) were within New York Heart Association (NYHA) functional class II-IV, (d) spoke or read Chinese, and (e) were willing to participate in this study, were recruited. Patients who (a) had serious comorbidities or mental disorders, (b) had severe hearing or visual impairments that meant they were unable to communicate, or (c) were unable to understand the questionnaires, were excluded. Written consent was obtained at the beginning of the investigation. To ensure the accuracy and integrity of the questionnaires, researchers checked the questionnaires after the patients finished completing them. Later, researchers collected patients' demographics and clinical characteristics by reviewing medical records or interviewing patients. Three hundred and twenty-one eligible participants with completed data were included in this study.

\subsection{Participants}

All of 321 patients in the original study were included in this study. The participants had a mean age of $63.6 \pm 10.6$ years. Of the 321 patients, nearly half $(48.6 \%)$ were female. The majority $(85.7 \%)$ of the patients were married or living with a partner, and $27.4 \%$ earned <1000 RMB (approximately 148 American dollars) monthly. In all, $63 \%$ of the patients were classified into NYHA functional class II, with a higher level of NYHA class, indicating more severe HF. The median of $\mathrm{N}$-terminal pro-B-type natriuretic peptide (NT-pro BNP) was $2384.6 \mathrm{pg} / \mathrm{mL}$. The details are shown in Table 1.

The target number of patients was 234 conducted by $\mathrm{G}^{*}$ Power software Version 3.1 based on a moderate $f^{2}$ effect size of $0.15, \alpha$ of 0.05 , and a power of $0.95 .{ }^{13}$ A total of 321 participants were enough for the sample size requirement in this study. 


\subsection{Measures}

\subsubsection{Depressive symptoms}

The Chinese version of the Depression Subscale of Hospital Anxiety and Depression Scale (HADS-D) was used to measure patients' depressive symptoms. ${ }^{14}$ This scale contains seven items that use a 4-point scale. The total score of this scale ranged from 0 to 21 , with a higher score indicating more severe depressive symptoms. Patients whose scores are $>7$ were considered to have depressive symptoms. The Chinese version of HADS-D is suitable for patients with HF, with Cronbach's $\alpha$ of $0.83 .{ }^{15}$ The Cronbach's $\alpha$ of HADS-D was 0.75 in our study.

\subsubsection{Functional capacity}

Functional capacity was measured using the Chinese version of the Duke Activity Status Index (DASI) in patients with HF. ${ }^{16}$ The DASI contains 12 items that assess whether the patients can do the activities. The total score for DASI was computed according to 12 items and their weighted scores. It ranges from 0 to 58.2, and a lower score indicates a poorer functional capacity. In HF patients, the Chinese version of DASI is reliable for HF patients, with Cronbach's $\alpha$ of $0.86 .{ }^{17}$ The Cronbach's $\alpha$ of DASI was 0.71 in our study.

\subsubsection{Social support}

Social support was assessed using the Chinese version of The Multidimensional Scale of Perceived Social Support (MSPSS)..$^{18}$ The MSPSS contains 3 dimensions and 12 items. Each item uses a 7-point scale. The total score of this scale ranges from 12 to 84 , and a lower score indicates a lower social support. The Chinese version of MSPSS is reliable for HF patients, with Cronbach's $\alpha$ of $0.91 .{ }^{19}$ The Cronbach's $\alpha$ of MSPSS was 0.84 in our study.

\subsubsection{Dispositional optimism}

The Chinese version of the Life Orientation TestRevised (LOT-R) scale was used to measure patients' dispositional optimism. ${ }^{20}$ The LOT-R contains 6 items which are rated on a 5-point Likert scale. According to the recommendation of scale developers, this study used this scale as a unidimensional scale. In this study, after deleting an invalid item, the total score ranges from 5 to 25 , with a lower score indicating less dispositional optimistic. The Chinese version of LOT-R is with Cronbach's $\alpha$ of $0.72 .{ }^{21}$ The Cronbach's $\alpha$ of LOT-R was 0.61 in our study.

\subsubsection{Self-care confidence}

Patients' self-care confidence was measured by the Chinese version of the subscale of self-care confidence in the Self-care of HF Index. ${ }^{22}$ The scale comprises six items with four options to assess patients' perceived confidence to engage in self-care behavior in the last month. The total score was standardized to a $0-100$ range, with a lower score indicating lower confidence in the ability to engage in self-care behaviors. The Chinese version of the subscale of self-care confidence in the Self-care of HF Index has been reported to be with Cronbach's $\alpha$ of $0.87 . .^{23}$ The Cronbach's $\alpha$ of the scale was 0.63 in our study.

\subsubsection{Health literacy}

The Chinese version of the Health Literacy Scale for Patients with Chronic Disease (HLS) was applied for assessing HF patients' health literacy. ${ }^{24}$ This scale was developed from Health Literacy Management Scale. ${ }^{25}$ The HLS consists of 24 items that assess the personal capacity to obtain, understand, and use health information. Each item uses a 5-point Likert scale. The total score for HLS ranges from 24 to 120 , and a lower score indicates poorer health literacy. HLS has been reported to be valid and reliable, and the Cronbach's $\alpha$ for HLS in Chinese patients ranges from 0.89 to $0.93 .{ }^{24}$ The Cronbach's $\alpha$ was 0.88 in our study.

\subsubsection{Demographic and clinical characteristics}

Demographic information included gender, age, education (<high school or $\geq$ high school), employment (unemployed or employed), marital status, residence (living in rural or urban area), and income $(<1000$ RMB or $\leq 1000$ RMB per month). Clinical characteristics included left ventricular ejection fraction $(<40 \%$ or $\geq 40 \%$ ), NYHA class, NT-pro BNP, previous hospitalizations due to $\mathrm{HF}$ ( $<3$ or $\geq 3$ times), disease duration (diagnosed with HF within 6 months or over 6 months), comorbidities $(<3$ or $\geq 3$ ), and body mass index (BMI). Demographics and clinical characteristics were collected through reviewing the medical record and interviewing the patient.

\subsection{Statistical analysis}

SPSS software for Windows version 20.0 (IBM Corp, Armonk, New York) was used for data analysis. Descriptive statistics were used to characterize the sample. Independent group $t$-tests and one-way analysis of variance were used to assess the difference in HADS-D score 
in demographic and clinical characteristics; Pearson correlation was used to assess the associations among continuous variables.

To assess the interaction effects, the interaction terms between potential stress and potential diatheses (DASI * MSPSS, DASI * LOT-R, DASI * self-care confidence, DASI * HLS) were generated. To control the effects of covariates on the study results, stepwise multiple linear regression with an entry criterion of $P<0.050$ and exit criterion of $P>0.100$ was used to assess the potential factors associated with HADS-D score. The independent variables included gender, age, education, employment, marital status, residence, income, left ventricular ejection fraction, NYHA class, NT-pro BNP level, previous hospitalizations due to $\mathrm{HF}$, disease duration, comorbidities, BMI, DASI, MSPSS, LOT-R, self-care confidence, HLS, DASI * MSPSS, DASI * LOT-R, and DASI * self-care confidence, DASI * HLS. A $P$-value of 0.050 was considered as significant.

\section{Results}

\subsection{Comparison of HADS-D score by sample characteristics}

In this study, 58 (18.1\%) patients had depressive symptoms. Higher HADS-D scores were found in patients whose education were less than high school $(P<0.001)$, who were unemployed $(P=0.018)$, who were single, divorced, or widowed $(P=0.046)$, who were living in an urban area $(P=0.002)$, whose income was $<1000 \mathrm{RMB}$ per month $(P=0.001)$, whose hospitalizations for HF were over three times $(P=0.002)$, and whose HF duration were over 6 months $(P=0.028)$. Age $(r=0.110, P=0.048)$ and NT-pro BNP level $(r=0.135$, $P=0.015)$ were positively correlated with HADS-D score. The HADS-D score had significant differences among NYHA class groups, and BMl groups. The details are shown in Table 1.

\subsection{Mean scores and correlations between potential influencing factors and HADS-D}

Mean scores for study variables and the correlation coefficients are shown in Table 2. The mean HADS-D score was $4.8 \pm 3.1$. Scores for functional capacity $(r=-0.353$, $P<0.001)$, social support $(r=-0.378, P<0.001)$, dispositional optimism $(r=-0.456, P<0.001)$, self-care confidence $(r=-0.321, P<0.001)$, and health literacy $(r=-0.462, P<0.001)$ were negatively correlated with the HADS-D score.

\subsection{Stepwise multiple linear regression analysis of HADS-D score}

A stepwise multiple linear regression identified that scores for functional capacity $(\beta=-0.043, P=0.007)$, social support $(\beta=-0.047, P=0.016)$, dispositional optimism $(\beta=-0.513, P<0.001)$, self-care confidence $(\beta=-0.032, P=0.002)$, and health literacy $(\beta=-0.076$, $P<0.001)$ were independently associated with the HADS-D score. In addition, the interactive term between scores for functional capacity and dispositional optimism $(\beta=0.017, P=0.007)$ was significant. This model accounted for $45.2 \%$ of the variance in HADS-D score. There was no multicollinearity issue in this study (tolerance was $>0.1$, and the variable inflation factor was $<10)$. The details are shown in Table 3.

\section{Discussion}

In the present study, we examined the possible factors associated with HF patients' depressive symptoms. Consistent with a previous study, we found that depressive symptoms were more prevalent in HF patients than in the general population. ${ }^{26}$ Functional capacity, social support, dispositional optimism, self-care confidence, and health literacy were negatively connected to depressive symptoms; these findings add to the existing literature.

In the present study, $58(18.1 \%)$ patients had depressive symptoms. In HF patients, there is a variation in the reported prevalence of depressive symptoms. Similar to our results, an observational study showed that $22.4 \%$ of HF patients had depressive symptoms. ${ }^{27}$ However, Fan and Meng ${ }^{28}$ reported that the prevalence was $44.1 \%$ in this population, which was higher. The differences in the prevalence across studies may be attributed to the differences in the severity of HF. Patients with more severe HF, as indicated by a higher NYHA class, have been reported to have a higher risk of depressive symptoms. ${ }^{29}$ The proportion of patients with an NYHA class of $\beta$ or $\chi$ was much higher $(55.6 \%)$ in the study by Fan and Meng than in this study (37.1\%) and in the study $(33.3 \%)$ by Graven et al. ${ }^{27}$ This may explain the relatively low prevalence in our study.

In this study, functional capacity was negatively related to depressive symptoms. Consistently, it was reported that functional capacity was related to HF patients' depressive symptoms ${ }^{30}$ Further support is provided by previous studies which suggested that exercise training improved HF patients' functional capacity and clinical outcomes, and reduced patients' depressive symptoms. ${ }^{31,32}$ Our finding adds evidence concerning the negative association between functional capacity 


\begin{tabular}{|c|c|c|c|c|c|c|c|}
\hline Variable & $\mathrm{N}(\%) / \mathrm{M} \pm \mathrm{SD} /$ Median $(\mathrm{Q})$ & $\begin{array}{l}\mathrm{HADS}-\mathrm{D} \\
\mathrm{M} \pm \mathrm{SD}\end{array}$ & $r$ & $t$ & F & $P$-value & Post hoc test \\
\hline Gender & & & & -0.064 & & 0.949 & \\
\hline Male & $165(51.4)$ & $4.8 \pm 3.2$ & & & & & \\
\hline Female & $156(48.6)$ & $4.8 \pm 3.1$ & & & & & \\
\hline Age, years & $63.6 \pm 10.6$ & & 0.110 & & & 0.048 & \\
\hline Education & & & & 4.002 & & $<0.001$ & \\
\hline$<$ High school & $209(65.1)$ & $5.3 \pm 3.0$ & & & & & \\
\hline 2High school & $112(34.9)$ & $3.9 \pm 3.2$ & & & & & \\
\hline Employment & & & & 2.383 & & 0.018 & \\
\hline Unemployed & $191(59.5)$ & $5.3 \pm 3.4$ & & & & & \\
\hline Employed & $130(40.5)$ & $4.5 \pm 2.9$ & & & & & \\
\hline Marital status & & & & 2.006 & & 0.046 & \\
\hline Single/divorced/widowed & $46(14.3)$ & $5.7 \pm 3.1$ & & & & & \\
\hline Married/cohabitating & $275(85.7)$ & $4.7 \pm 3.1$ & & & & & \\
\hline Residence & & & & 3.049 & & 0.002 & \\
\hline Rural area & $123(38.3)$ & $5.5 \pm 3.4$ & & & & & \\
\hline Urban area & $198(61.7)$ & $4.4 \pm 2.9$ & & & & & \\
\hline Income (RMB per month) & & & & 3.318 & & 0.001 & \\
\hline$<1000$ & $88(27.4)$ & $5.8 \pm 3.5$ & & & & & \\
\hline 1000 & $233(72.6)$ & $4.5 \pm 2.9$ & & & & & \\
\hline LVEF & & & & 0.789 & & 0.431 & \\
\hline$<40 \%$ & $51(15.9)$ & $5.1 \pm 3.4$ & & & & & \\
\hline$\geq 40 \%$ & $270(84.1)$ & $4.8 \pm 3.1$ & & & & & \\
\hline NYHA class & & & & & 6.590 & 0.002 & \\
\hline (a) II & 202 (62.9) & $4.4 \pm 3.0$ & & & & & $a<b^{\star *}, a<c^{\star *}$ \\
\hline (b) III & $93(29.0)$ & $5.5 \pm 3.2$ & & & & & $b<c$ \\
\hline (c) IV & $26(8.1)$ & $6.0 \pm 3.5$ & & & & & \\
\hline NT-pro BNP, pg/mL & $2384.6(262.3-2714.1)$ & & 0.135 & & & 0.015 & \\
\hline Hospitalizations & & & & -3.187 & & 0.002 & \\
\hline$<3$ & $261(81.3)$ & $4.6 \pm 3.2$ & & & & & \\
\hline$\geq 3$ & $60(18.7)$ & $6.0 \pm 2.7$ & & & & & \\
\hline HF duration & & & & -2.206 & & 0.028 & \\
\hline$<6$ months & $202(62.9)$ & $4.5 \pm 3.2$ & & & & & \\
\hline$\geq 6$ months & $119(37.1)$ & $5.3 \pm 2.9$ & & & & & \\
\hline Comorbidities & & & & -0.945 & & 0.345 & \\
\hline$<3$ & $39(12.1)$ & $4.7 \pm 3.1$ & & & & & \\
\hline$\geq 3$ & $282(87.9)$ & $5.1 \pm 3.1$ & & & & & \\
\hline$B M I, k g / m^{2}$ & & & & & 6.371 & $<0.001$ & \\
\hline (a) $<18.5$ & $11(3.4)$ & $7.7 \pm 3.1$ & & & & & $a>b^{*}, a>c^{* *}, a>d^{* \star *}$ \\
\hline (b) 18.5-23.9 & $86(26.8)$ & $5.5 \pm 3.5$ & & & & & $b>c^{*}, b>d^{* *}$ \\
\hline (c)24.0-27.9 & $147(45.8)$ & $4.6 \pm 3.0$ & & & & & $c>d$ \\
\hline (d) ${ }^{3} 28.0$ & $77(24.0)$ & $4.1 \pm 2.5$ & & & & & \\
\hline
\end{tabular}

Note: BMI, body mass index; HADS-D, Depression Subscale of Hospital Anxiety and Depression Scale; HF, heart failure; LVEF, left ventricular ejection fraction; NYHA, New York Heart Association; NT-proBNP, N-terminal pro B type natriuretic peptide.

${ }^{\star} P<0.050 ;{ }^{* \star P}<0.010 ;{ }^{\star \star \star} P<0.001$.

Table 1. Comparison of HADS-D score by sample characteristics. 


\begin{tabular}{|c|c|c|c|c|c|c|c|}
\hline Variable & Mean & $\mathrm{SD}$ & 1 & 2 & 3 & 4 & 5 \\
\hline 1. HADS-D & 4.8 & 3.1 & 1 & & & & \\
\hline 2. DASI & 20.6 & 9.3 & $-0.353^{\star \star \star}$ & 1 & & & \\
\hline 3. MSPSS & 64.7 & 7.4 & $-0.378^{\star \star \star}$ & $0.170^{\star *}$ & 1 & & \\
\hline 4. LOT-R & 20.7 & 2.2 & $-0.456^{\star \star \star}$ & $0.110^{*}$ & $0.325^{\star \star *}$ & 1 & \\
\hline $\begin{array}{l}\text { 5. Self-care } \\
\text { confidence }\end{array}$ & 57.1 & 13.4 & $-0.321^{\star \star \star}$ & $0.126^{*}$ & $0.212^{* * *}$ & $0.156^{\star *}$ & 1 \\
\hline 6. HLS & 99.5 & 12.5 & $-0.462^{\star \star \star}$ & $0.335^{\star * *}$ & $0.275^{\star * *}$ & 0.107 & $0.299 * * *$ \\
\hline
\end{tabular}

Note: DASI, Duke Activity Status Index; HADS-D, Depression Subscale of Hospital Anxiety and Depression Scale; HLS, Health Literacy Scale for Patients with Chronic Disease; LOT-R, Life Orientation Test-Revised; MSPSS, Multidimensional Scale of Perceived Social Support.

${ }^{\star} P<0.050 ;{ }^{* *} P<0.010 ;{ }^{* \star *} P<0.001$.

Table 2. Mean scores and correlation coefficients between potential influencing factors and HADS-D $(n=321)$.

\begin{tabular}{|c|c|c|c|c|c|c|c|}
\hline \multirow{2}{*}{ Variables } & \multirow{2}{*}{$\beta$} & \multirow{2}{*}{\multicolumn{2}{|c|}{$95 \% \mathrm{Cl}$}} & \multirow{2}{*}{$t$} & \multirow{2}{*}{$P$-value } & \multicolumn{2}{|c|}{ Collinearity statistics } \\
\hline & & & & & & Tolerance & VIF \\
\hline Hospitalizations <3 & 0.737 & 0.039 & 1.436 & 2.079 & 0.038 & 0.878 & 1.139 \\
\hline DASI & -0.043 & -0.075 & -0.012 & -2.734 & 0.007 & 0.778 & 1.285 \\
\hline MSPSS & -0.047 & -0.086 & -0.009 & -2.426 & 0.016 & 0.806 & 1.241 \\
\hline LOT-R & -0.513 & -0.639 & -0.388 & -8.035 & $<0.001$ & 0.863 & 1.159 \\
\hline Self-care confidence & -0.032 & -0.053 & -0.012 & -3.092 & 0.002 & 0.859 & 1.164 \\
\hline HLS & -0.076 & -0.099 & -0.053 & -6.503 & $<0.001$ & 0.785 & 1.273 \\
\hline DASI*LOT-R & 0.017 & 0.005 & 0.030 & 2.723 & 0.007 & 0.934 & 1.071 \\
\hline
\end{tabular}

Note: Dependent variable: HADS-D score. Adjusted $R^{2}=0.452$. Gender, age, education, employment, marital status, residence, income, left ventricular ejection fraction, NYHA class, NT-pro BNP, disease duration, comorbidities, BMI, and DASI * MSPSS, DASI * self-care confidence, DASI * HLS were excluded by stepwise multiple linear regression.

$\beta$, standardized beta; BMI, body mass index; CI, confidence interval: DASI, Duke Activity Status Index; HLS, Health Literacy Scale for Patients with Chronic Disease; HADS-D, Depression Subscale of Hospital Anxiety and Depression Scale; LOT-R, Life Orientation Test-Revised; MSPSS Multidimensional Scale of Perceived Social Support; NYHA, New York Heart Association; NT-proBNP, N-terminal pro B type natriuretic peptide; VIF, variance inflation factor.

Table 3. Stepwise multiple linear regression analysis of HADS-D score $(n=321)$.

and depressive symptoms, and indicates that preventing HF patients' functional capacity from decreasing may be helpful to relieve depressive symptoms in HF patients.

Our study showed that HF patients with lower social support had more severe depressive symptoms. Similarly, in a review of 15 studies, 11 studies showed a consistent result that lower social support was significantly related to HF patients' depressive symptoms. ${ }^{33}$ Family members are key sources of social support for Chinese HF patients. However, elderly patients with HF may lack support from their young family members due to the changes in society and economy in China, wherein young people relocate to more developed cities for work or study, leaving their older family members at home. ${ }^{34}$ Our result suggests that improving HF patients' social support be a potential target of intervention to alleviate depressive symptoms.

In this study, low dispositional optimism was related to depressive symptoms in patients with HF. Similarly, two other studies showed that optimism was significantly related to depressive symptoms in the general population and depressive population. ${ }^{26,35}$ Our study adds evidence to confirm the negative relationship between dispositional optimism and depressive symptoms in patients with HF. As a group-based optimism training program has been proved to be beneficial to cardiac patients, ${ }^{36}$ improving dispositional optimism may reduce depressive symptoms in HF patients.

This study showed that HF patients with a lower level of self-care confidence had more severe depressive symptoms. Similarly, it was reported that interventions that self-care education could reduce depressive symptoms in patients with thalassemia. ${ }^{37}$ Our study provides a novel view that HF patients' low self-care confidence is important in the development of depressive symptoms. The present study suggested that interventions aimed at promoting HF patients' self-care confidence, such as motivational interviewing or cognitive behavioral interventions, may be beneficial for reducing their depressive symptoms. 
We found that lower health literacy was related to more severe depressive symptoms in HF patients. Our finding was supported by a meta-analysis of 23 studies, ${ }^{38}$ which revealed that health literacy was correlated with mental quality of life. The current study expands our understanding that low health literacy may be a potential diathesis to develop depressive symptoms when patients confront HF. Strategies, such as providing health-related comic books, focused on improving health literacy may contribute to relieving HF patients' depressive symptoms.

It is worth noting the interaction effect between functional capacity and dispositional optimism on HF patients' depressive symptoms. Our study indicated that HF patients with lower dispositional optimism may have more depressive symptoms when their functional capacity decreased. To our knowledge, this is the first study to examine the interaction effect on HF patients' depressive symptoms, which adds evidence to the existing literature.

Several limitations of this study should be recognized. First, the current study was a secondary data analysis of a cross-sectional study, the primary purpose of which was not to explore the possible factors associated with depressive symptoms. Perhaps choices were limited to the factors measured in the original study. For instance, treatment for depression was not available, but it was a covariate in this study. Further intervention studies are warranted to confirm the causality. Second, in our sample, most of the patients were classified as NYHA class II, which may limit the potential to generalize our results to the entire HF population. Future studies need to be conducted with a more representative sample. Third, this study used self-reported measures.

\section{References}

1. Lin X, Gao B, Huang J. Prevalence of depressive symptoms in patients with Heart Failure in China: a meta-analysis of comparative studies and epidemiological surveys. JAffect Disord. 2020;274:774-783.

2. Guck TP, Elsasser GN, Kavan MG, Barone EJ. Depression and congestive heart failure. Congestive Heart Fail (Greenwich, Conn.). 2003;9:163-169.

3. Koenig HG. Depression in hospitalized older patients with congestive heart failure. Gen Hosp Psychiatry. 1998;20:29-43.

4. Sbolli M, Fiuzat M, Cani D, O'Connor CM. Depression and heart failure: the lonely comorbidity. Eur J Heart Fail. 2020;22:2007-2017.

5. Patel N, Chakraborty S, Bandyopadhyay D, et al. Association between depression and readmission
Thus, potential reporting bias may threaten the evidence of our findings, despite the good reliability and validity of the instruments.

Our study makes sense for clinical practice because it provides referential information for the development of interventions to relieve HF patients' depressive symptoms. Our findings suggest that healthcare providers should take these factors, including functional capacity, social support, dispositional optimism, self-care confidence, and health literacy, into consideration to develop effective interventions to relieve their depressive symptoms.

\section{Conclusions}

In conclusion, as many HF patients have depressive symptoms, healthcare providers should pay attention to early detection and management of depressive symptoms to improve HF patients' outcomes. Moreover, impaired functional capacity, low social support, low dispositional optimism, low self-care confidence, and low health literacy were associated with more severe depressive symptoms in patients with HF. Therefore, interventions targeted at improving the above factors may result in a reduction of depressive symptoms in HF patients.

\section{Ethical approval}

Ethical issues are not involved in this paper.

\section{Conflicts of interest}

All contributing authors declare no conflicts of interest. of heart failure: a national representative database study. Prog Cardiovasc Dis. 2020;63:585-590.

6. Sherwood A, Blumenthal JA, Trivedi R, et al. Relationship of depression to death or hospitalization in patients with heart failure. Arch Intern Med. 2007;167:367-373.

7. Sherwood A, Blumenthal JA, Hinderliter AL, et al. Worsening depressive symptoms are associated with adverse clinical outcomes in patients with heart failure. J Am Coll Cardiol. 2011;57: 418-423.

8. Jiang W, Kuchibhatla M, Cuffe MS, et al. Prognostic value of anxiety and depression in patients with chronic heart failure. Circulation. 2004;110:3452-3456. 
9. Carney RM, Howells WB, Blumenthal JA, et al. Heart rate turbulence, depression, and survival after acute myocardial infarction. Psychosom Med. 2007;69:4-9.

10. Schowalter M, Gelbrich G, Stoerk S, et al. Generic and disease-specific health-related quality of life in patients with chronic systolic heart failure: impact of depression. Clin Res Cardiol. 2013;102:269-278.

11. Monroe SM, Simons AD. Diathesis stress theories in the context of life stress research - implications for the depressive-disorders. Psychol Bull. 1991;110:406-425.

12. Hankin BL. Adolescent depression: description, causes, and interventions. Epilepsy Behav. 2006;8:102-114.

13. Faul F, Erdfelder E, Buchner A, Lang A. Statistical power analyses using $G^{*}$ Power 3.1: tests for correlation and regression analyses. Behav Res Methods. 2009;41:1149-1160.

14. Zigmond AS, Snaith RP. The hospital anxiety and depression scale. Acta Psychiat Scand. 1983;67:361-370.

15. Chen C, Fang W, An Y, Wang L, Fan X. The multiple mediating effects of illness perceptions and coping strategies on the relationship between physical symptoms and depressive symptoms in patients with heart failure. Eur J Cardiovasc Nurs. 2020;19:125-133.

16. Hlatky MA, Boineau RE, Higginbotham MB, et al. A brief self-administered questionnaire to determine functional-capacity (the duke activity status index). Am J Cardiol. 1989;64:651-654.

17. Fan X, Lee KS, Frazier SK, Lennie TA, Moser DK. Psychometric testing of the Duke Activity Status Index in patients with heart failure. Eur J Cardiovasc Nurs. 2015;14:214-221.

18. Zimet GD, Powell SS, Farley GK, Werkman S, Berkoff KA. Psychometric characteristics of the multidimensional scale of perceived social support. J Pers Assess. 1990;55:610-617.

19. Fan X, LV F. Psychosocial factors associated with self-efficacy for managing chronic disease in patients with chronic heart failure. Eur J Cardiovasc Nurs. 2016;15:255-261.

20. Scheier MF, Carver CS, Bridges MW. Distinguishing optimism from neuroticism (and trait anxiety, self-mastery, and self-esteem) - a reevaluation of the life orientation test. J Pers Soc Psychol. 1994;67:1063-1078.

21. Liu B, Pu J, Hou H. Effect of perceived stress on depression of Chinese "Ant Tribe" and the moderating role of dispositional optimism. $J$ Health Psychol. 2016;21:2725-2731.
22. Riegel B, Lee CS, Dickson VV, Carlson B. An update on the self-care of heart failure index. J Cardiovasc Nurs. 2009;24:485-497.

23. Kang X, Himmelfarb CRD, Li Z, et al. Construct validity of the chinese version of the self-care of heart failure index determined using structural equation modeling. J Cardiovasc Nurs. 2015;30:222-228.

24. Sun HL, Peng H, Fu H. Dimensional structure of health literacy scale for patients with chronic disease. J Environ Occup Med. 2013;3:171-175.

25. Jordan JE, Buchbinder R, Osborne RH. Conceptualising health literacy from the patient perspective. Patient Educ Couns. 2010;79:36-42.

26. Bonsaksen T, Grimholt TK, Skogstad L, et al. Selfdiagnosed depression in the Norwegian general population - associations with neuroticism, extraversion, optimism, and general self-efficacy. BMC Public Health. 2018;18:1076.

27. Graven LJ, Martorella G, Gordon G, Keltner JSG, Higgins MK. Predictors of depression in outpatients with heart failure: an observational study. Int J Nurs Stud. 2017;69:57-65.

28. Fan X, Meng Z. The mutual association between depressive symptoms and dyspnea in Chinese patients with chronic heart failure. Eur J Cardiovasc Nurs. 2015;14:310-316.

29. Celik E, Cay S, Sensoy B, et al. Heart failure functional class associated with depression severity but not anxiety severity. Acta Cardiol Sin. 2016;32:55-61.

30. Saleh ZT, Wu J, Salami I, Yousef K, Lennie TA. The association between depressive symptoms and N-Terminal Pro-B-Type natriuretic peptide with functional status in patients with heart failure. J Cardiovasc Nurs. 2018;33:378-383.

31. Das A, Roy B, Schwarzer G, et al. Comparison of treatment options for depression in heart failure: a network meta-analysis. J Psychiatr Res. 2019;108:7-23.

32. O'Connor CM, Whellan DJ, Lee KL, et al. Efficacy and safety of exercise training in patients with chronic heart failure HF-ACTION randomized controlled trial. JAMA. 2009;301:1439-1450.

33. Graven LJ, Grant J. The impact of social support on depressive symptoms in individuals with heart failure update and review. J Cardiovasc Nurs. 2013;28:429-443.

34. Chen $Y$, Hicks A, While AE. Loneliness and social support of older people in China: a systematic literature review. Health Soc Care Community. 2014;22:113-123.

35. Kube T, Siebers VHA, Herzog P, et al. Integrating situation-specific dysfunctional expectations and dispositional optimism into the cognitive model of 
depression - a path-analytic approach. J Affect Disord. 2018;229:199-205.

36. Mohammadi N, Aghayousefi A, Nikrahan GR, et al. A randomized trial of an optimism training intervention in patients with heart disease. Gen Hosp Psychiatry. 2018;51:46-53.

37. Allahyari J, Masinaeinezhad N, Abdollahimohammad A, Moghadam MP, Rahnama
M. The effect of self-care education based on the Orem's model on depression in patients with B-Thalassemia major: a semi-experimental study. Med Sci. 2018;22:52-57.

38. Zheng $M$, Jin $H$, Shi $N$, et al. The relationship between health literacy and quality of life: a systematic review and meta-analysis. Health Qual Life Outcomes. 2018;16:201. 